\title{
On MHD rotational transport, instabilities and dynamo action in stellar radiation zones
}

\author{
S. Mathis ${ }^{1,2}$, J.-P. Zahn ${ }^{2}$ and A.-S. Brun ${ }^{1,2}$ \\ ${ }^{1}$ Laboratoire AIM, CEA/DSM-CNRS-Université Paris Diderot, IRFU/SAp, \\ F-91191 Gif-sur-Yvette Cedex, France \\ email: stephane.mathis@cea.fr, allan-sacha.brun@cea.fr \\ ${ }^{2}$ LUTH, Observatoire de Paris-CNRS-Université Paris Diderot, \\ 5 Place Jules Janssen, F-92195 Meudon Cedex, France \\ email: jean-paul.zahn@obspm.fr
}

\begin{abstract}
Magnetic field is an essential dynamical process in stellar radiation zones. Moreover, it has been suggested that a dynamo action, sustained by a MHD instability which affects the toroidal axisymmetric magnetic field, could lead to a strong transport of angular momentum and of chemicals in such regions. Here, we recall the different magnetic transport and mixing processes in radiative regions. Next, we show that the dynamo cannot operate as described by Spruit (2002) and recall the condition required to close the dynamo loop. We perform highresolution 3D simulations with the ASH code, where we observe indeed the MHD instability, but where we do not detect any dynamo action, contrary to J. Braithwaite (2006). We conclude on the picture we get for magnetic transport mechanisms in radiation zones and the associated consequences for stellar evolution.
\end{abstract}

Keywords. MHD, Sun: magnetic fields, Sun: interior, stars: magnetic fields, stars: interiors

\section{MHD instabilities and possible dynamo in stellar radiation zones}

Purely axisymmetric poloidal and toroidal fields are unstable (see Pitts \& Tayler 1985 and references therein). Moreover, Spruit (2002) suggests that the instability of such toroidal field could sustain a dynamo in stellar radiation zones. This idea is quite interesting, but we argue that this dynamo cannot operate as he describes it. According to him, the non-axisymmetric instability-generated small-scale field, which has zero average, is wound up by the differential rotation "into a new contribution to the azimuthal field. This again is unstable, thus closing the dynamo loop." But this shear induced azimuthal field has the same azimuthal wavenumber as the instability-generated field, i.e. $m \neq 0$ and predominantly $m=1$ : it has no mean azimuthal component, and thus it cannot regenerate the mean toroidal field that is required to sustain the instability. For the same reason, the instability-generated field cannot regenerate the mean poloidal field, as was suggested by Braithwaite (2006). Therefore, the Pitts \& Tayler instability cannot be the cause of a dynamo, as it was described by Spruit and Braithwaite. In fact, the dynamo loop can only be achieved through the azimuthal average of the fluctuation-fluctuation term of the induction equation $\left\langle\vec{\nabla} \times\left(\vec{v}^{\prime} \times \vec{B}^{\prime}\right)>_{\varphi}\right.$ (cf. Zahn, Brun \& Mathis 2007).

\section{Numerical simulations}

We perform 3D-numerical simulations of the problem using the global ASH code (Clune et al. 1999, Brun et al. 2004) to solve the relevant anelastic MHD equations in a spherical shell representing the upper part of the solar radiation zone $\left(0.35 \leqslant r / R_{\odot} \leqslant 0.70\right)$ using a resolution of $N_{r} \times N_{\theta} \times N_{\varphi}=193 \times 128 \times 256$. A detailed discussion of the set-up is 


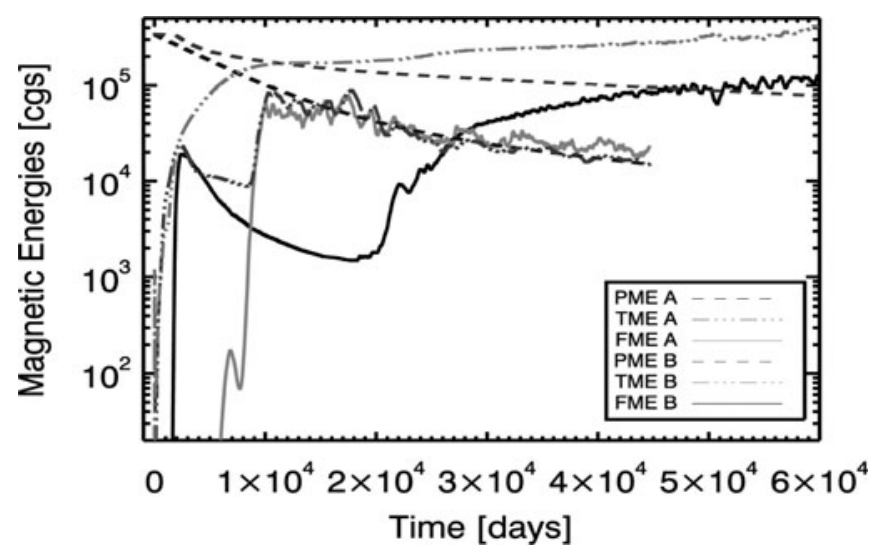

Figure 1. Time evolution of the energies of the mean poloidal (PME), mean toroidal (TME) and non-axisymmetric (FME) components of the magnetic field. Cases A and B refer respectively to higher and lower magnetic diffusivity. Note the steady decline of the poloidal field, which is not affected by the irruption of the $m=1$ Pitts \& Tayler instability (at $\mathrm{t} \approx 8,000$ days in case $\mathrm{A}$ and $\approx 20,000$ days in case B). (Zahn, Brun \& Mathis 2007, courtesy A\&A)

given in Brun \& Zahn (2006) and in Zahn, Brun \& Mathis (2007). We study the case A discussed in Brun \& Zahn (2006) and we performed an additional series of simulations with a lower Ohmic diffusivity (by a factor of 10, case B), in order to reach a higher magnetic Reynolds number in favor of a dynamo. In our simulations (cf. Fig. 1), the $\alpha$-effect plays a negligible role since no regeneration of the mean poloidal field is found, at least up to the magnetic Reynolds number $R m=R^{2} \Delta \Omega / \eta \sim 10^{5}$ for Prandtl number $P_{m}=\nu / \eta=1$. On the other hand, the $\beta$-effect, i.e. the turbulence-enhanced diffusivity, is absent here. Hence, one should not expect much mixing of the stellar material and the magnetic transport of angular momentum is mainly due to the Lorentz torque that leads to Ferraro's law $(\vec{B} \cdot \vec{\nabla} \Omega=0)$ (cf. Brun \& Zahn 2006). In fact, the smallest resolved scales do not act on the mean poloidal field as a turbulent diffusivity: they seem to behave rather as gravito-Alfvén waves. Finally, there is no sign either of a small-scale fluctuation dynamo. To check this point, we suppressed the mean poloidal field at the latest stage of our simulation. Then, the mean toroidal field decreases rapidly, because it is no longer produced by the $\Omega$-effect, and the instability-generated field accompanies its decline. Thus the fluctuating field does not maintain itself. Therefore, we conclude that in our simulations the Pitts \& Tayler instability is unable to sustain a large-scale mean field dynamo, in the parameter domain that we have explored.

\section{References}

Braithwaite, J. 2006, A\& A 449, 451

Brun, A.-S. \& Zahn, J.-P. 2006, A\&A 457, 665

Brun, A.-S., Miesch, M. S., \& Toomre, J. 2004, ApJ 614, 1073

Clune, T. L. et al. 1999, Parallel Comput. 25, 361

Mathis, S. \& Zahn, J.-P. 2005, A\&A 440, 653

Pitts, E. \& Tayler, R. J. 1985, MNRAS 216, 139

Spruit, H. C. 2002, A\&SA 381, 923

Zahn, J.-P., Brun, A.-S., \& Mathis, S. 2007, A $\& A 474,145$ 